\section{FIGURE FEMMINILI NEI RACCONTI DI ANTONIO}

\section{TABUCCHI}

WOMEN IN ANTONIO TABUCCHI'S TALES

Zosi Zografidou

Universidad de Salónica

RIASSUNTO:

Nelle opere di Tabucchi leimmagini femminili In Tabucchi's works, women are really assumono una particolare importanza important for the development of the plot nell'evoluzione della trama. Sono ritratte nella They are represented in their individuality and loro individualità ed ognuna di esse si adatta all of them adapts to the social environment all'ambiente sociale in cui vive. La donna è where she lives. Women are a silent presence, una presenza silenziosa, isolata e dinamica they are silent, isolated and dynamic. They e assume il ruolo di figlia, sorella, moglie, can take the role of daughter, sister, wife, e assume il ther sife,

\section{PAROLE CHIAVE:}

KEY WORD:

Donna, opera, Tabucchi.

Woman, work, Tabucchi.
I libri di Tabucchi conducono attraverso le parole e le varie voci ad un incrocio tra realtà e fantasia, tra vita reale e sogno, ci trascinano lontano in un viaggio verso altre terre, ci fanno sognare ad occhi aperti, con gli occhi dell'anima e cifanno infine riscoprire il valore delle capacità creative della nostra immaginazione.Il tempo che fugge, che scorre, è un tema ricorrente e prediletto intutte le opere dello scrittore, come nel libro Si sta facendo sempre piùtardi (Tabucchi, 2003) anche ne Il tempo invecchia in fretta(Tabucchi, 2005).In questo contesto, in un viaggio «fuori dal tempo e dallo spazio» (Tabucchi, 2010: 15),in uno spazio senza limiti, si svolgono le storie e si snodano le vicende.

I personaggi che agiscono in queste storie sono creature che possono essere interpretate come specchio di immagini femminili del novecento, della società odierna. Sono donne che si muovono nella quotidianità, sono persone, come definisce Tabucchi, «sempre alla ricerca di qualche cosa» (Gambaro, 2013), che vivono in silenzio tra di noi, senza essere osservati, ? assumendo anche particolare importanza nella evoluzione della trama narrativa.

«Sono uomini o donne», dice Di Paolo, «che inciampano nei casi e negli equivoci dell'esistere, e che attraversano slanci passionali, amori travolgenti, emozioni vere o presunte, rancori e nostalgie» (Di Paolo, 2003). Ma sono anche persone morte che vengono da un altro mondo, che parlano, che appaiono in sogno, che ritornano misteriosamente vive per trasportarci in un altrove e farci partecipi lettori della sua opera (p.e.: Tabucchi, 1991: 29; Tabucchi, 2005: 109; Tabucchi, 2006: 122).

Sull'argomento ha detto Tabucchi:

Viaggiando si incontrano soprattutto i vivi. A volte anche dei moribondi. E anche dei veri morti, dipende dai luoghi. Oggi in certi paesi, ad esempio, se ne può trovare una quantità ragguardevole. Ma anche i nostri morti, o i morti che abbiamo conosciuto quando erano vivi. Può capitare. Può capitare, per esempio, che in una modesta pensione di Lisbona, in una domenica d'agosto, quando la città è deserta, alcuni esempi tratti dall' opera di Tabucchi. examples are taken from Tabucchi's work. uno riceva la visita del proprio padre morto da tempo. (Tabucchi, 2010:15)

Marcello Cella in una intervista a Tabucchi del 1995 ha chiesto allo scrittore sui personaggi femminili e sulla loro complicità con il protagonista. «Si tratta di un ruolo in qualche modo subordinato o di una scelta espressiva di altro tipo?» Lo scrittore ha risposto:

$-\ll$ Non so se è subordinato. Credo che per uno scrittore uomo sia molto difficile affrontare la tematica femminile, il mondo femminile. Scrittori che hanno saputo affrontare, secondo me con molta efficacia, il mondo femminile si contano sulle dita. A me viene in mente Faubert con Karenina. E non sono scrittori che si trovano tutti i giorni». (Cella, 2013)

\begin{tabular}{|c|c|c|}
\hline $\begin{array}{l}\text { madre, amante, serva. Vengono presentat } \\
\text { alcuni esempi tratti dall' opera di Tabucchi. }\end{array}$ & $\begin{array}{l}\text { mother, lover or servant. In this article, some } \\
\text { examples are taken from Tabucchi's work. }\end{array}$ & uno riceva la visita del proprio padre morto da tempo. (Tabucchi, 2010: 15) \\
\hline PAROLE CHIAVE: & KEY WORD: & $\begin{array}{l}\text { Marcello Cella in una intervista a Tabucchi del } 1995 \text { ha chiesto allo scrittore sui } \\
\text { personaggi femminili e sulla loro complicità con il protagonista. «Si tratta di un ruolo }\end{array}$ \\
\hline Donna, opera, Tabucchi. & Woman, work, Tabucchi. & $\begin{array}{l}\text { in qualche modo subordinato o di una scelta espressiva di altro tipo?» Lo scrittore ha } \\
\text { risposto: }\end{array}$ \\
\hline & & $\begin{array}{l}\text { - «Non so se è subordinato. Credo che per uno scrittore uomo sia molto difficile } \\
\text { affrontare la tematica femminile, il mondo femminile. Scrittori che hanno saputo } \\
\text { affrontare, secondo me con molta efficacia, il mondo femminile si contano sulle } \\
\text { dita. A me viene in mente Flaubert con Madame Bovary e Tolstoj con Anna } \\
\text { Karenina. E non sono scrittori che si trovano tutti i giorni». (Cella, 2013) }\end{array}$ \\
\hline
\end{tabular}


Tabucchi parlando del suo romanzo epistolare Si sta facendo sempre più tardi dice che è difficile fare la parte della donna per uno scrittore uomo e pertanto si limita a scrivere nell'opera lettere inviate solo da uomini. Nonostante queste parole, nella sua opera i personaggi femminili sono numerosi e hanno sempre un peso nel caratterizzare la società, assumono un ruolo più importante di quanto possa sembrare a prima vista $\mathrm{e}$ di quanto lo scrittore ci voglia far credere. Accanto a essi stiamo sempre a cercare e a trovare un uomo, un ragazzo, un giovane. Sarebbe posssibile il Gioco del rovescio senza Maria do Carmo Meneses de Sequeira? O I pomeriggi di Sabat? senza Nenna, lamamma o la zia Yvonne? O "Il penoso caso del signor Silva da Silva e Silva" senzala vecchia e fedele cameriera Maria da Piedade de Lourdes da Ascencao e la giovane Maria de Fatima Samantha, l'ultima camerierina arrivata in casa Silva da Silva e Silva? Certamente la risposta sarebbe negativa.

Le immagini femminili sono tutte figure delineate nella loro individualità, con una varietà di sfumature che prendono varie forme e rivestono ruoli importanti. Sono voci d'oltretomba, voci del passato, voci di un'antica quotidianità, voci interiori che si incontrano e si rispondono a vicenda nel più profondo dei racconti. Delle volte nella sua opera i personaggi sono assenti, sono fantasmi o esseri immaginati e allo stesso momento sono presenti. Come le donne inesistenti e immaginarie nelle lettere scritte da uomini in Si sta facendo sempre più tardi o Miriam, la contessa du Terrail,in Rebus dei Piccoli equivoci senza importanza, sognata indossando «una lunga veste bianca che da lontano sembrava una camicia da notte» (Tabucchi, 1991: 29). Quando mi è passata vicino mi ha investito una folata di aria gelida, come un alone
che si portava dietro: e allora, con lo stupore senza sorpresa dei sogni, ho capito che era morta [...] (Tabucchi, 1991: 29)

Ma poi domani, o un altro giorno, sognerò che Miriam è viva, essa passerà vicino al mare e acconsentira al mio richiamo e si siedera vicino a me su una sdraii della spiaggia di Biarritz, o un'altra idea di spiaggia, si ravvierà i capelli come faceva lei, con un gesto lento e languido, pieno di sensi, e guardando il mare mi indichera con un gesto lent, entrambi, di esserci trovati al nostro appuntamento. (Tabucchi, 1991: 29)

Nel percorso del viaggio in treno del protagonista de il Gioco del rovescio verso il Portogallo, fatto come tante altre volte nella sua vita, nella luce di Lisbona «bianca verso la fose e rosata sulle coline» che dopo la notte diventa 'fonda', nel «crepuscolo che stava calando sulla città» quando «si accendevano le prime luci», «le luci che si spensero di nuovo», in tutti questi posti descritti prima, appaiono immagini di Maria do Carmo, che era morta. L'assenza provoca in lui «una sensazione strana, come se dall' alto stessi a guardare un altro se stesso che in una notte di luglio, dentro lo scompartimento di un treno semibuio, stesse entrando in un paese straniero per andare a vedere una donna che conosceva bene e che era morta». Con il nome di questa donna imprescindibile nel testo comincia uno dei racconti più conosciuti di Antonio Tabucchi.

Quando Maria do Carmo Meneses de Sequeira morì, io stavo guardando Las Meninas di Velazquez al museo del Prado. Era un mezzogiorno di luglio e io non sapevo che lei stava morendo. Restai a guardare il quadro fino alle dodici e un quarto, poi uscii lentamente cercando di trasportare nella memoria l'espressione della figura di fondo, ricordo che pensai alle parole di Maria do Carmo; la chiave del quadro sta nella figura di fondo, è un gioco del rovescio. (Tabucchi, 1981: 9)

Forse - dice Maria do Carmo- sei troppo giovane per capire, alla tua età io non avrei capito, non avrei immaginato che la vita fosse come un gioco che giocavo nella mia infanzia a Buenos Aires. (Tabucchi, 1981: 11)

Nell'opera di Tabucchi le cose sono viste e interpretate attraverso il loro rovescio. Il rovescio per Tabucchi non è solo una parola «casualmente capitata nel testo», è una parola che sottende un universo di pensiero che ne struttura l'attività narrativa. L'autore nella prefazione della seconda edizione scrive di essersi accorto che «una certa cosa che era così era invece anche in un altro modo» e questa affermazione ha segnato la sua produzione letteraria successiva (Tabucchi, 1988: 5).

Antonio Tabucchi, studioso anche di Pessoa, per bocca di Maria do Carmo che diventa portavoce dello scrittore e della sua filosofia, esprime la sua inquietudine per la vita e mostra la sua opinione su Pessoa definito da lui «il genio perchè ha capito il risvolto delle cose, del reale e dell'immaginato» (Tabucchi, 1981: 11).

Lei mi prendeva la mano -ricorda il protagonista- e mi diceva: senti, chissà cosa siamo, chissà dove siamo, chissà perchè ci siamo, senti, viviamo questa vita come se fosse un revès, per esempio stanotte, tu devi pensare che sei me e che stai stringendo te fra le tue braccia, io penso di essere te che sto stringendo me fra le mie braccia. (Tabucchi, 1981: 15)

La Saudade, diceva Maria do Carmo, non è una parola, è una categoria dello spirito, solo i portoghesi riescono a sentirla perchè hanno questa parola per dire che ce l'hanno, lo ha detto un grande poeta. E allora cominciava a parlare di Fernando Pessoa. (Tabucchi, 1981: 10)

Maria sente il suo rovescio che ha la sua duplicità. Raccontava di essere figlia di esiliati (Tabucchi, 1981:13) con «un'infanzia infelice» mentre il marito Nuno Meneses de Sequeira afferma che era figlia di grandi proprietari e che aveva avuto un'infanzia dorata essendo anche la donna più corteggiata di Lisbona (Tabucchi, 1981: 21).

In albergo aprii la lettera. Su un foglio bianco c'era scritta, in stampatello e senza accenti, la parola SEVER. La rovesciai meccanicamente, in pensiero, e poi sotto, 
anch'io in stampatello e senza accenti, scrissi con la matita: REVES. Meditai un attimo su quella parola ambigua, che poteva essere spagnola o francese e aveva due significati assolutamente diversi. (Tabucchi, 1981: 23)

In spagnolo 'REVES' significa rovescio, mentre in francese 'sogni'.

E questo gioco del rovescio, la protagonista lo ha giocato per tutta la vita con la consapevolezza del marito, raggiungendo il rovescio forse con la sua morte... morte che è molto presente nell'opera di Tabucchi.

In Lettera da Casablanca, del libro Il gioco del rovescio ci si imbatte nella sorella Lina, nel fratello Ettore che racconta di diventare Giosefine in una lunga lettera da Casablanca, in Carmen de Rio che cantava in maniera divina, nella madre che «si sedeva al piccolo pianoforte che testimoniava della sua educazione, di una giovinezza agiata, di un padre cancelliere» (Tabucchi, 1981: 28) e anche nella zia Olga che «non era cattiva, era una veneta ciarliera e brontolona che era rimasta caparbiamente attaccata al suo dialetto» e quando «parlava non si capiva quasi, mescolava il veneto con lo spagnolo» (?abucchi, 1981: 31).

Ne I pomeriggi di Sabato de Il gioco del rovescio è presente la sorella Nena che portava «un apparecchio di metallo sui denti superiori», che aveva un gatto rossiccio, «il mio Belafonte» come lo chiamava, che giocava con le sue bambole, «le sue amichette», disposte sull'aiuola che serviva da pavimento (Tabucchi, 1981: 78); appaiono anche la zia Yvonne «con le sue lunghe gonne di seta, camicette di chiffon, il capellino elegante con la camelia di organza» (Tabucchi, 1981: 81) e la mamma che «a volte piangeva in silenzio con la testa reclinata» (Tabucchi, 1981: 85), che passeggiava per la casa mettendo in ordine il nulla che c'era da mettere in ordine, spostando un soprammobile di qualche centimetro (Tabucchi, 1981: 87). Amelia delle Stanze dei Piccoli equivoci senza importanza guarda:

il leggero velo di nebbia che in lontananza sta calando sul tetto della casa e pensa: è tardi, dobbiamo affrettarci. Il sentiero è ripido e sinuoso, lastricato di granito tagliato largo, con l'umidità della sera sembra un ruscello pietrificato dal tempo ...

(Tabucchi, 1991: 63)

Nelle storie di Tabucchi i personaggi femminili saltano fuori da paesaggi pittoreschi sfilano nell'intreccio e nello scorrere del tempo. In questo spazio fuori del tempo incontriamo le immagini femminili che si accordano con l'ambiente sociale nel quale vivono. Sono presenze silenziose, isolate e dinamiche che assumono pluralità di ruoli: la donna diventa figlia, sorella, moglie, madre, amante, serva ... suscitandoci sentiment di simpatia, di dolore, di nostalgia, di felicità e provocando parole e pensieri sulla vita e sulla morte.

\section{RIFERIMENTI BIBLIOGRAFICI}

AA.VV., Antonio Tabucchi narratore, Atti della giornata di studi (17 novembre 2006), a cura di Silvia Contarini e Paolo Grossi, Parigi, Istituto Italiano di Cultura, 2007.

Di Paolo, P., La fine di qualcosa. Scrittori italiani tra due secoli, Roma, Perrone, 2012.

Di Paolo, P., Dacia Maraini, Romana Petri, Ugo Riccarelli, Una giornata con Tabucchi, con intervista di Carlos Gumpert, lo scrittore raccontato dai suoi amici, Roma, Cavallo di ferro, 2012.

Ladron de Guevara, P. L., «Il passato e altrove: Spazio versus tempo ne Il tempo invecchia in fretta di Antonio Tabucchi», in Tempo spazio e memoria nella letteratura italiana. Omaggio a Antonio Tabucchi, a cura di Zosi Zografidou, Università 'Aristotele' di Salonicco, Dipartimento di Lingua e Letteratura Italiana, Roma-Salonicco, ARACNE - USPress, pp.402-410, 2012.

Rimini, T., Album Tabucchi. L'immagine nelle opere di Antonio Tabucchi, Palerno, Sellerio, 2011.

Tabucchi, A., Il gioco del rovescio, 1a ed., Milano, il Saggiatore, 1981.

---- Il gioco del rovescio, 2a ed., Milano, Feltrinelli, 1988.

---- Piccoli equivoci senza importanza, Milano, Feltrinelli, 1991.

---- Si sta facendo sempre più tardi. Romanzo in forma di lettere, Milano, Feltrinelli, 2003.

--- Racconti, Milano, Feltrinelli, 2005.

---- Il tempo invecchia in fretta, Milano, Feltrinelli, 2005.

---- Tristano muore, Milano, Feltrinelli, 2006.

---- Viaggi e altri viaggi, a cura di Paolo di Paolo. Milano, Feltrinelli, 2010.

---- Di tutti resta un poco. Letteratura e cinema, a cura di Anna Dolfi. Milano, Feltrinelli, 2013.

\section{CITAZIONI INTERNET:}

Cella, Marcello, "Intervista ad Antonio Tabucchi", in http://www.neokifilm.it /articoli/ intervista-ad-antonio-tabucchi (data di consultazione: 16/4/2013).

Di Paolo, Paolo, “La letteratura come memoria: il corpo, la nostalgia, il caso: attraverso l'opera del grande scrittore Antonio Tabucchi" (Milano, 10 febbraio 2003) in http://www.italialibri. net/dossier/tabucchi/memoria.html (data di consultazione: 9/1/2011).

Gambaro, Fabio, “Conversazione con Antonio Tabucchi”, in http://www. feltrinellieditore.it/ SchedaTesti?id_testo=1468\&id_int=1384 (data di consultazione: 10/5/2013). 